\title{
Interactive comment on "Compilation of pollen productivity estimates and a taxonomically harmonised PPE dataset from Northern Hemisphere extratropics" by Mareike Wieczorek and Ulrike Herzschuh
}

Anonymous Referee \#2

Received and published: 2 April 2020

Synthesis of relative pollen productivity estimates (RPP) is useful to achieve pollen-based quantitative reconstructions of plant cover for the purpose of palaeoenvironmental and -climate studies taking plant cover into consideration. RPP is one of the most important parameters in the models of quantitative vegetation reconstruction (e.g. REVEALS and LOVE model, Sugita, 2007a and b). The reliability of RPP determines the reliability of the vegetation reconstruction. Therefore, it is important to check the theory and methodology behind each original publication to include only reliable pollen productivity before calculating a mean of such values. My major concern of this 
study is that it does not take into consideration of earlier evaluation of RPPs (Mazier et al., 2012 for Europe; Li et al., 2018 for temperate China), which is not good for the ESSDD reliability of future quantitative reconstruction if the unreliable RPP values are used. My second concern is, so far there is no test about whether the RPPs of one continent are reliable for application in quantitative reconstruction of another continent available so far, so it is better to handle them separately. With the reasons mentioned above, I would recommend a major revision.

I suggest following revising strategy:

1.List all available relative pollen productivity estimates, indicate the ones that evaluated by experts or tested for reliability in the original publication.

2.Check the reliability of each study through following steps:

1) There are several assumptions behind the ERV model, the reliability of the RPP values depend on whether the assumptions of the ERV model in the study are meet, check each study and keep the ones meet the assumptions.

2) In theory, log-likelihood will increase as the distance from sampling site increases and gradually reach an asymptote at the distance of relevant source area of pollen (RSAP). Check and keep only the studies with theoretically correct log-likelihood against distance curve.

3) Check the SE and RPP, retain the ones that $S E<R P P$

4) The RPPs from different continents can be very different mainly due to different plant species involved for same pollen type. Test of the reliability of sharing the RPPs among continents with observations (e.g. Hellman et al., 2008, Journal of Quaternary Science) or historical vegetation maps (Cui et al., 2014, Ecology and Evolution) is very important, but will be very time consuming and difficult to collect such data, therefore no such tests available so far. It is therefore important to prepare the RPP dataset of each continent separately for this study.

Interactive

comment 
5) Calculate the mean of the retain values from above and do box plot of the PPEmeans by excluding values defined as values outside the range of \pm 1.5 interquartilerange for each continent separately.

3. It is important to warn the readers the importance of using only the reliable RPPs.

4. In the dataset file, please do not mix the ERV sub-models and dispersion functions, they are totaly different things. Please indicate the distance weighting method of each study.

To achieve the goal of a more constructive and useful dataset of this synthesis for future application in quantitative reconstruction, I would recommend a second review of revised version by experts in ERV model and quantitative vegetation reconstruction models.

Interactive comment on Earth Syst. Sci. Data Discuss., https://doi.org/10.5194/essd-2019-242, 2020. 